\title{
EVALUATION OF ANTI-FUNGICIDAL DRUGS IN THE TREATMENT OF BALB/C MICE INOCULATED WITH Lacazia loboi.
}

THESIS. P. S. Rosa submitted this thesis for her Doctorate in Tropical Diseases at Botucatu School of Medicine, São Paulo State University, UNESP, Botucatu, São Paulo, Brasil, 2003.

Advisor: Professor Diltor Vladimir Araujo Opromolla

ABSTRACT: The objective of this study was to test the murine model, BALB/c mice inoculated with Lacazia loboi for therapeutic assays using the fungus viability index, number of fungi, histopathological examination, and appearance of microscopic lesions as evaluation parameters at different post-treatment times. Itraconazol, terbinafine, and terbinafine formulated with $\beta$-cyclodextrin were the anti-fungal drugs tested. One hundred and twenty-eight BALB/c mice were intradermally inoculated with L. Ioboi into both hind footpads. The mice were divided into four groups of 32 each. Treatment started 45 days post-inoculation and lasted 13 months. Drugs were orally administered mixed with powdered commercial feed. Itraconazol was used at the dose of $4 \mathrm{mg} / \mathrm{kg}$ and terbinafine at $5 \mathrm{mg} / \mathrm{kg}$. Eight animals in each group were randomly sacrificed four, seven, ten, and fifteen months after inoculation. Each time, both footpads were clinically evaluated for the development of macroscopic lesions and then excised for histopathological examination, viability index determination, and fungal count. All groups showed a progressive increase in the number of fungi throughout the experiment, which suggests fungal multiplication in the footpads. Although there was no statistical difference between the groups, the lowest number of fungi was in the terbinafine and itraconazol-treated groups. In relation to viability index, a significant difference was found $(p<0.05)$ between the terbinafine-treated groups and the control ten months after inoculation; the same did not happen in the itraconazol-treated group. All groups started 
to show macroscopic alterations after seven months of inoculation. The number of animals that developed lesions did not vary between the control and treated mice. Histopathological examination showed a granulomatous infiltrate consisting predominantly of histiocytes and giant cells containing large numbers of fungi, many with characteristics of non-viable fungal cells. It was not possible to distinguish between treated and non-treated animals by the lesion histopathological aspects. An evident action of the drugs used in this mouse model could not be observed, except for the effect of terbinafine on $L$. loboi, demonstrated by the low viability in the group treated with this drug compared with the control. Several factors can explain these results: drug characteristics and pharmacokinetics; doses; administration route; fungus biochemical structure; or inherent factor to the mouse model. However, BALB/c mice showed to be a suitable model for further studies in this area.

KEY WORDS: Jorge Lobo's Disease, treatment; itraconazol, terbinafine.

CORRESPONDENCE TO: P. S. Rosa, Rua Prof. Cristino Cabral, 2-13, Jardim Santana, 17020-240, Bauru, São Paulo, Brasil. Phone: 5514 2261790. Mobile: 551497115506. Email: sammarco@hotmail.com 